Ann. Biol. anim. Bioch. Biophys., I969, 9 (4), 497-512.

\title{
RESTAURATION PAR LA THYROXINE DE LA CROISSANCE DE LA LAINE SUPPRIMEE LORS DE L'HYPOPHYSECTOMIE CHEZ DES BREBIS ILE-DE-FRANCE
}

\author{
J. GIRARD \\ avec la collaboration technique de Liliane LEGAULT \\ Station centrale de Physiologie animale, \\ Centre national de Recherches zootechniques, 78 - Jouy-en-Josas \\ Institut national de la Recherche agronomique
}

\section{SOMMAIRE}

La croissance pondérale de la laine, sa vitesse de croissance en longueur, son diamètre et la morphologie de la surface de la cuticule du poil de laine, ont été étudiés chez des Brebis Ile-de-France au cours de trois phases expérimentales :

- avant hypophysectomie,

- après hypophysectomie sans supplémentation hormonale, à l'exclusion d'une dose de I $2,5 \mathrm{mg} / \mathrm{j}$ de cortisone pour assurer la survie des animaux,

- après hypophysectomie et administration de la L-thyroxine aux doses de $1,25^{-6,25}-12,5^{\circ}$ et $40,0 \mu \mathrm{g} / \mathrm{kg} / \mathrm{j}$.

Lorsque l'hypophysectomie est parfaite, c'est-à-dire lorsqu'il ne subsiste aucune cellule hypophysaire, il y a pratiquement arrêt de la croissance de la laine au bout de 4 semaines et tous les follicules pileux producteurs de laine entrent en repos, selon un processus habituel aux follicules pileux à fonctionnement cyclique (stade télogène).

L'administration de la $\mathrm{L}$-thyroxine a restauré à elle seule les différents paramètres de la croissance de la laine dans leur intégralité.

\section{INTRODUCTION}

FERGUSON, WALIACE et LINDNER ont montré, en I965, que la croissance de la laine, qui est permanente chez le Mouton, est complètement arrêtée au bout de 4 à 26 semaines après 1'hypophysectomie : cette croissance de la laine peut être rétablie et même augmentée, par rapport à la croissance normale, à la suite d'administration de thyroxine.

Il reste cependant, en s'appuyant sur les expériences de ces auteurs, à préciser :

Annales de Biologie animale. - 1969. 
I. Quelle forme revêt, après l'hypophysectomie, l'arrêt du fonctionnement des follicules pileux de la toison : stade télogène classique des follicules pileux à fonctionnement cyclique, ce qui est tout à fait exceptionnel pour la laine, ou forme particulière, étant donné la nature de l'intervention qui a provoqué l'arrêt du fonctionnement des follicules pileux.

2. Si les variations pondérales, constatées par FERGuSON à la suite de l'hypophysectomie et de l'action consécutive de l'administration de la thyroxine, affectent également les trois paramètres de la production de la laine, c'est-à-dire le nombre de follicules pileux en activité, la vitesse de croissance en longueur et le diamètre, ou certains d'entre eux seulement. On peut, en effet, se poser cette question, car on sait que l'action des hormones thyroïdiennes ne porte que sur la vitesse de croissance en longueur de la laine et pas du tout sur le diamètre (THERIEZ et ROUGEO', I962).

3. Enfin, si ces variations de croissance affectent la structure de la laine, car il a été ainsi prouvé que les hormones thyroïdiennes modifiaient la surface de la cuticule (ROUGEOT, I965).

\section{MATÉRIEL ET MÉTHODES}

\section{Les animaux}

Les observations ont été faites sur I 8 Brebis Ile-de-France dont la toison n'est composée que de laine, à l'exclusion de tout autre type de poil à croissance cyclique.

Ces brebis, ni gestantes, ni allaitantes, étaient âgées de $\mathbf{I}$ à 2 ans au début de l'expérience pendant laquelle elles furent maintenues dans des conditions constantes de température $\left(23^{\circ} \pm \mathrm{I}^{\circ} \mathrm{C}\right)$, d'humidité ( $60 \pm$ Io p. I00 H. R.) et d'alimentation ( $85^{\circ} \mathrm{g} / \mathrm{jour} /$ Brebis d'un mélange de concentré de farine de luzerne et de céréales et de la paille donnée ad libitum). Les animaux ont été pesés tous les 14 jours.

Après 3 mois d'observations préliminaires, les Brebis furent hypophysectomisées, leur poids étant compris à ce moment entre 43 et $55 \mathrm{~kg}$.

\section{Hypophysectomie et traitements}

\section{I. Hypophysectomie.}

L'hypophysectomie a été réalisée par la voie parapharyngée, selon une technique mise au point par DenamuR et MARTINeT (communjcations personnelles). Nous avons délibérément laissé en place, pour ne point léser l'hypothalamus, la partie de la tige pituitaire accolée à l'éminence médiane.

La survie des animaux a été assurée après l'hypophysectomie par un traitement à l'acétate de cortisone (Sovetal), injecté par voie intramusculaire, à la dose de $12,5 \mathrm{mg} /$ jour/Brebis, qui, tout en étant suffisante, était théoriquement assez faible pour ne pas avoir d'action inhibitrice sur la croissance de la laine (FRASER et SHORT, I960).

\section{2. Administration de la L-thyroxine.}

L'administration de la L-thyroxine (Calbiochem) eut lieu au moins un mois après que la croissance de la laine fut complètement arrêtée ou, tout au moins, fut descendue à un palier assez bas (20 \pm 8 p. 100 en poids de la valeur initiale) à la suite de l'hypophysectomie (tabl. I et fig. 4).

$\mathrm{La}$ L-thyroxine, dissoute dans du liquide physiologique amené à $\mathrm{pH} \mathrm{IO}_{2}$ par addition de $\mathrm{NaOH}$ et tamponné $\left(\mathrm{CO}_{3} \mathrm{HNa}-\mathrm{CO}_{3} \mathrm{Na}_{2}\right)$, fut injectée par voie sous-cutanée aux doses de $1,25,6,25,12,5^{\circ}$ et $40,0 \mu \mathrm{g} / \mathrm{kg} /$ jour.

\section{3. Controle de l'hypophysectomie.}

Toutes les Brebis ont été abattues à la fin de l'expérience en vue de vérifier histologiquement par le trichrome de frabe s'il ne subsistait pas des cellules hypophysaires fonctionnelles. 


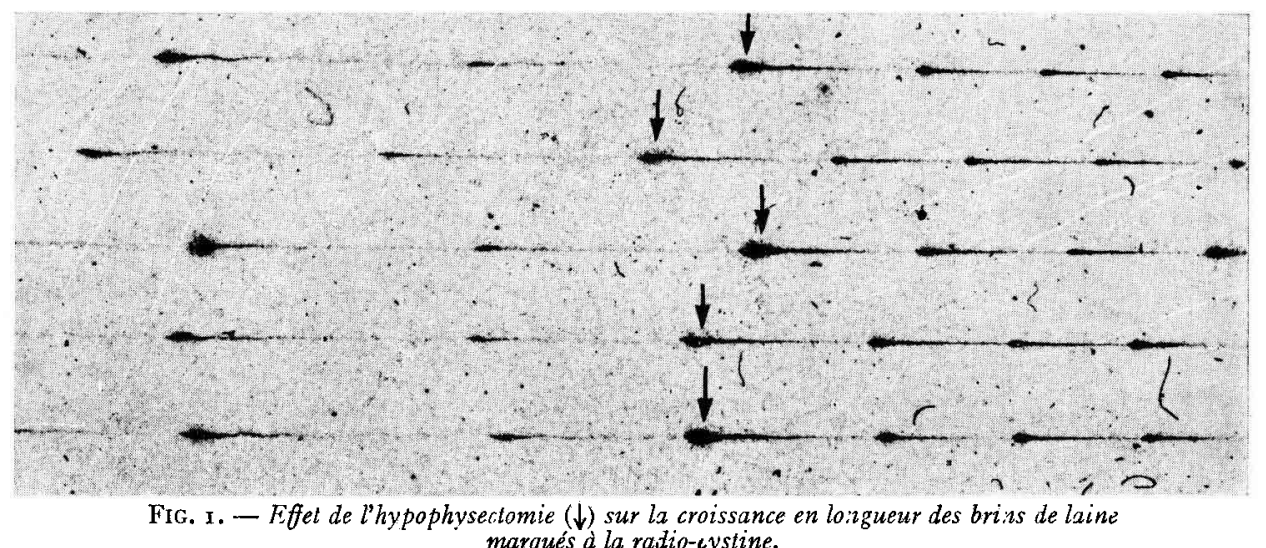
marqués à la radio-cystine.

A gauche de la flèche $(\downarrow)$ croissance normale avant l'hypophysectomie, à droite de la flèche on observera la réduction importante de la vitesse de croissance en longueur après l'hypophysectomie (intervalle entre deux points : croissance durant 14 jours)

\section{Mesures de la croissance de la laine}

\section{3. $\mathrm{r}$. Croissance pondérale de la laine.}

Afin de suivre les variations de la croissance de la laine en cours d'expérience, nous avons prélevé tous les 14 jours, par rasage, la laine d'une aire de $40 \mathrm{~cm}^{2}$ limitée par tatouages et située sur le dos. Ces échantillons de laine ont été lavés et dégraissés avant d'être pesés.

\section{2. Examen histologique de la peau.}

L'évolution de l'activité des follicules pileux et les variations de leur densité ont été suivis par des prélèvements de peau, effectués sur le flanc à l'aide d'un emporte-pièce circulaire de $\mathrm{r} \mathrm{cm}^{2} \mathrm{de}$ section, tous les I 4 jours, ou de manière plus rapprochée pour la période immédiatement aprèsl'hypophysectomie $(3,5,7$, to et I4 jours). Après avoir été fixés au Bouin alcoolique (20 p. roo éthanol à $95 \mathrm{p}$. I0o), ces prélèvements furent coupés à $7 \not<$ et colorés successivement au rouge solide, à l'orange $G$ et au bleu d'aniline. Cette coloration permet de distinguer aisément au niveau des glandes sébacées, les follicules pileux en activité ou stade anagène (fibre orangée, gaine externe du follicule pileux grisâtre), des follicules pileux au repos ou au stade télogène (fibre vermillon, gaine externe bleutée) (fig. 2 et 3 ).

Le pourcentage des follicules pileux en télogène avant et après l'hypophysectomie et lors du traitement à la L-thyroxine, a été déterminé au microscope, au grossissement de 125 , à l'aide d'un réticule oculaire quadrillé ; les intervalles de confiance de la détermination des pourcentages ont été donnés par les tables de Fisher et YaTES (tabl. r).

Comme il est impossible de prélever des morceaux de peau ayant la même surface, on ne peut s'assurer par un simple comptage de la disparition éventuelle des follicules pileux après l'hypophysectomie. C'est pour cela que nous nous sommes adressés au rapport du nombre de follicules pileux secondaires au nombre de follicules pileux primaires, S/P défini par CARTER (I943) : en effet, les follicules pileux primaires sont des éléments fixes de la peau et sont facilement repérables par la présence d'un canal de la glande sudoripare qui leur est adjacent (fig. 2 et 3 ), ainsi toute variation du S/P reflète une variation de la population des follicules pileux (tabl. 2).

Le dénombrement des follicules pileux primaires et secondaires a été fait par projection microscopique au grossissement de $5^{\circ}$,

\section{3. Mesure de la vitesse de croissance en longueur.}

Elle fut effectuée en marquant la laine de repères radioactifs datés, obtenus à l'aide d'injections sous-cutanées de cystine ${ }^{35} \mathrm{~S}$ (C. E. A.) faites tous les I4 jours (ROUGEOT, I959, I962). Les injections furent pratiquées sur 2 repères tatoués sur la peau du dos et situés à ro $\mathbf{c m}$ de part et d'autre de l'épine dorsale au niveau de la dernière côte. A la fin de l'expérience, soit 14 jours après la dernière injection, les mèches de laine radioactives furent prélevées à proximité immédiate des tatouages. L'autoradiographie de 3 o brins de laine, pris au hasard dans chaque mèche et étendus rectilignement 


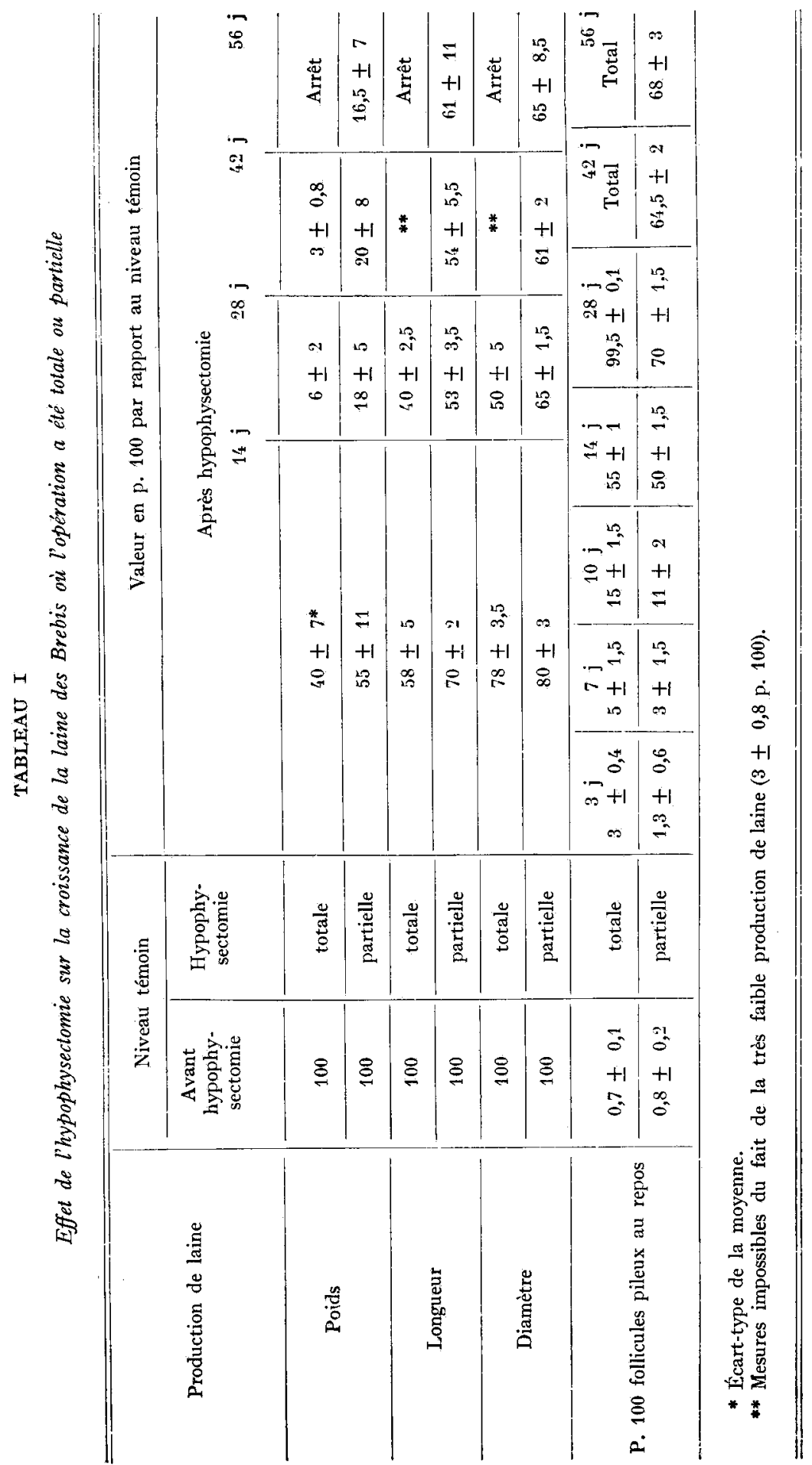


sur des lames de verre, a été observée sur un film rayons X (Kodirex) appliqué fortement contre eux pendant 2 mois. La mesure des intervalles séparant les repères radioactifs successifs (fig. I) a été faite par projection microscopique au grandissement linéaire de 20.

\section{4. Mesure des diamètres.}

Les diamètres ont été mesurés sur 1o des 30 brins de laine utilisés pour les autoradiographies. A cet effet, nous avons placé les autoradiographies sous les lames de verre en faisant coincider le brin de laine à son image dans le film ; ensuite, nous avons reporté à l'encre, par transparence, les repères radioactifs sur les brins de laine eux-mêmes. Dix diamètres ont été ainsi mesurés, à l'aide du grossissement 500 (Visopan de Reichert), pour chaque intervalle compris entre 2 points radioactifs successifs.

\section{5. Morphologie de la surface de la cuticule.}

Pour rendre compte des modifications morphologiques provoquées par les divers traitements sur la laine, nous avons observé, sur les brins utilisés pour la mesure des diamètres, les changements de la surface de la cuticule. Pour cela, nous avons compté en suivant une parallèle à l'axe de la fibre, le nombre de rebords d'écailles compris entre 2 points radioactifs successifs : nous avons ainsi établi, d'une part, le nombre de rebords d'écailles par $\mathrm{mm}$ de laine et, d'autre part, le nombre de rebords d'écailles produits par jour (tabl. 3).

\section{RÉSULTATS}

\section{Action de l'hypophysectomie}

\section{I. Contrôle de l'hypophysectomie.}

Le contrôle histologique des selles turciques a montré qu'il n'existait plus aucune cellule hypophysaire, ni de tissu hypophysaire dégénéré chez ro Brebis sur I8. Chez celles-ci, la production de laine cessa complètement après 1'hypophysectomie : à partir du $28^{\mathrm{e}}$ jour pour 6 d'entre elles et $\mathrm{du} 4^{\mathrm{e}}$ jour pour les 4 suivantes (ex. fig. 4).

Par contre, chez les 8 autres Brebis où nous avons observé la présence d'îlots hypophysaires fonctionnels, la croissance pondérale de la laine s'est maintenue, à partir du $28 \mathrm{e}$ jour après l'hypophysectomie, à $20 \pm 8 \mathrm{p}$. Ioo du niveau initial (ex. fig. 4) ; chez 5 Brebis, cette croissance s'est poursuivie avec quelques fluctuations irrégulières jusqu'à la fin de l'expérience (I2 à r6 semaines), alors que chez les 3 autres, elle s'arrêta au bout de 14, I 8 ou 24 semaines.

\section{2. Arrêt de l'activité des follicules pileux.}

Chez les ro Brebis où 1'hypophysectomie a été complète, l'examen histologique de la peau a révélé que la mise en repos des follicules pileux producteurs de laine s'établissait progressivement $(3 \pm 0,4$ p. Ioo à 3 jours, $5 \pm I, 5$ p. I0o à 7 jours, I5 $\pm \mathrm{I}, 5 \mathrm{p}$. Ioo à Io jours et $55 \pm \mathrm{I}$ p. Ioo à $\mathrm{I} 4$ jours) pour devenir totale entre le $28^{\mathrm{e}}$ jour et le $4^{\mathrm{e}}$ jour (tabl. I). Cet arrêt total de la croissance de la laine est définitif (cas d'une Brebis conservée dans cet état pendant I an).

Chez les 8 Brebis où l'ablation de la glande fut incomplète, nous avons assisté également à une mise en repos des follicules pileux : à I 4 jours le taux $(50 \pm 1,5 \mathrm{p}$. Ioo est encore semblable à celui des Brebis dont 1 'hypophysectomie a été totale $(55 \pm \mathrm{I}$ p. roo); par contre, à 28 jours, nous n'avons compté que $70 \pm I, 5$ p. Ioo de follicules pileux au repos, au lieu de $99,5 \pm 0, I$ p. Ioo et, par la suite, ce taux de 70 p. Ioo s'est maintenu autour de cette valeur avec des fluctuations irrégulières (tabl. I). 


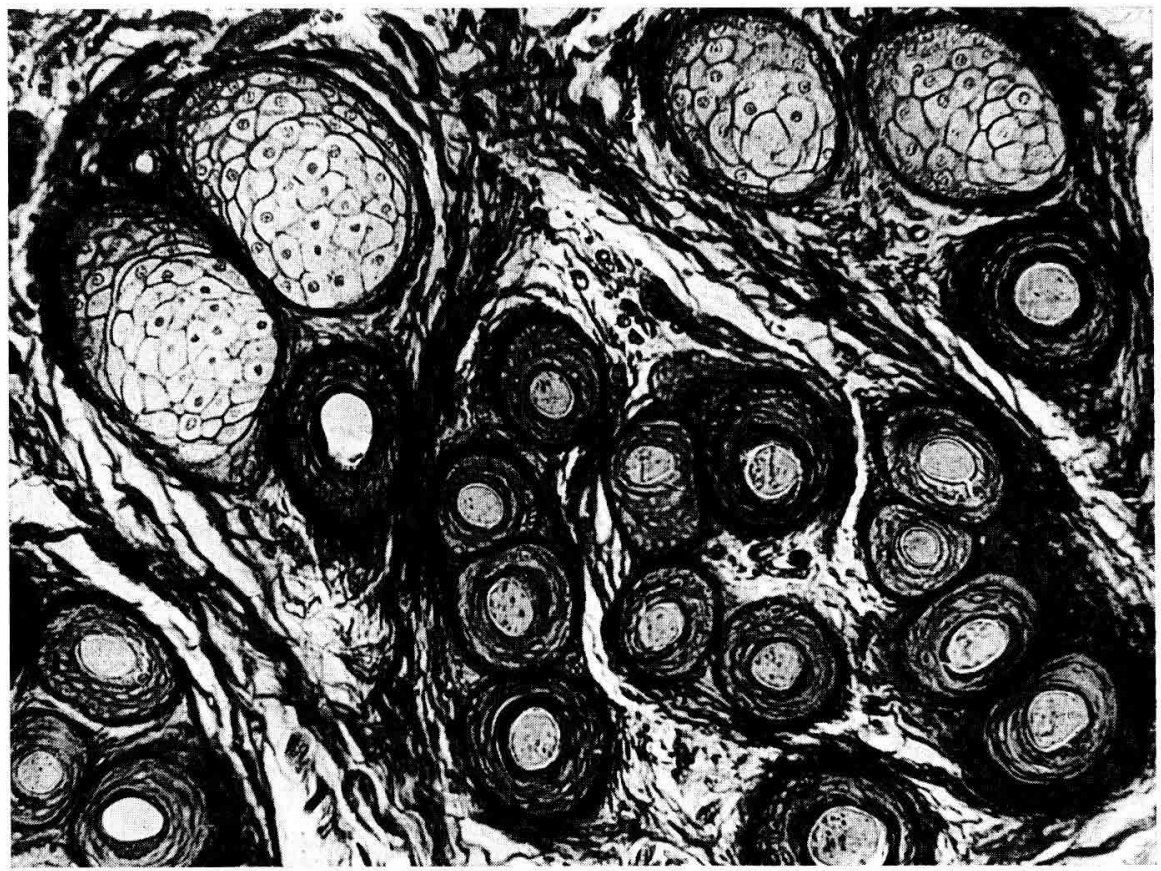

FIG. 2. - Follicules pileux en activitè chez une Brebis normaile

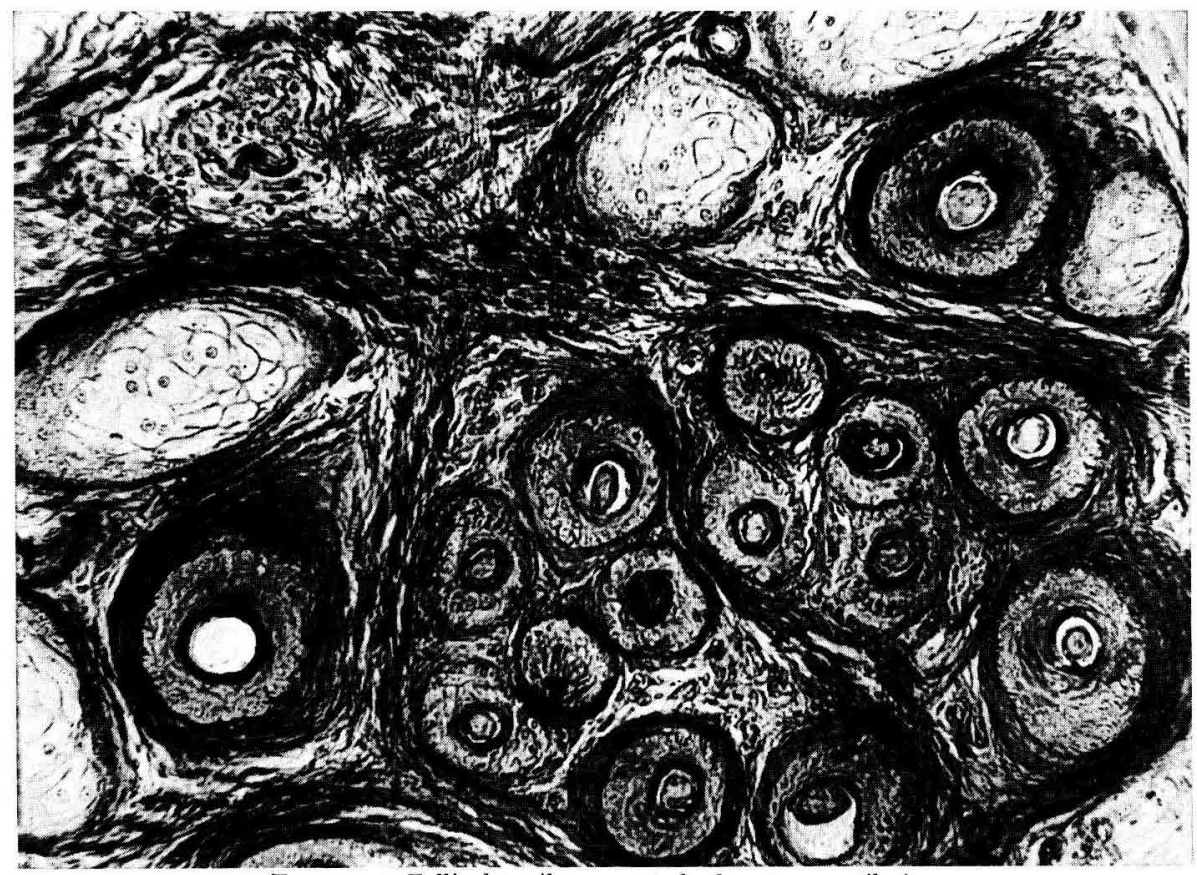

FIG. 3. - Follicules pilieux au stade de repos ou télogène chez la même Brebis 42 jours après l'hypophysectomie 
On peut donc admettre que si l'activité des follicules pileux n'a pas été totalement arrêtée à 28 jours (ou presque, avec seulement $0,5 \mathrm{p}$. Ioo de follicules pileux en activité), c'est que 1'hypophysectomie n'a pas été parfaitement réalisée.

Les follicules pileux qui ont cessé leur activité, présentent l'image du stade de repos normal des follicules pileux à activité cyclique, le télogène (fig. 3) : racine du poil fibrillaire montrant la présence de groupements sulfhydriles (réaction de BARRNETT et SELIGMAN) et englobée dans une capsule de tissu conjonctif dont les noyaux des cellules sont orientés radialement. Il s'agit donc d'une évolution du follicule pileux vers le stade de repos normal et non d'une atrophie quelconque, comme le supposent FERGUSON et al. (I965).

Enfin, l'examen histologique de la peau n'a pas révélé de modifications de la structure des groupes folliculaires (fig. 3 à comparer avec la fig. 2) : les glandes sudoripares, les glandes sébacées ct les muscles arrecteurs sont tous présents, ainsi que tous les follicules pileux, d'après les mesures du $\mathbf{S} / \mathbf{P}$ (tabl. 2).

TABLEAU 2

Valeur du rapport SIP chez des Brebis dont l'hypophysectomie a été totale ou partielle

\begin{tabular}{|c|c|c|c|c|c|c|c|}
\hline & \multirow{2}{*}{$\begin{array}{c}\text { Avant } \\
\text { hypophy- } \\
\text { sectomie }\end{array}$} & \multirow{2}{*}{$\begin{array}{l}\text { Hypo- } \\
\text { physec- } \\
\text { tomie }\end{array}$} & \multicolumn{5}{|c|}{ Après hypophysectomie } \\
\hline & & & $14 \mathrm{j}$ & $28 \mathrm{j}$ & $42 \mathrm{j}$ & $56 \mathrm{j}$ & Thyroxine \\
\hline \multirow{2}{*}{ Rapport S/P } & $8,8 \pm 0,3^{*}$ & totale & $7,5 \pm 0,2$ & $7,8 \pm 0,2$ & Arrêt & Arrêt & $8,0 \pm 0$, \\
\hline & $8,0 \pm 0,5$ & partielle & $7,5 \pm 0,4$ & $7,0 \pm 0,3$ & $7,0 \pm 0,2$ & $7,8 \pm 0,5$ & $7,6 \pm 0,4$ \\
\hline
\end{tabular}

* Écart-type de la moyenne.

\section{3. Action sur la croissance et la morphologie de la laine.}

Chez toutes les Brebis où l'hypophysectomie a été totale, les mesures de la croissance en longueur, du diamètre ainsi que l'examen de la surface de la cuticule ont été faits sur les quelques brins de laine dont la croissance avait persisté jusqu'au $28^{e}$ jour après l'hypophysectomie : en effet, les brins de laine qui avaient continué à croître jusqu'au $42^{\mathrm{e}}$ jour (4 Brebis) étaient trop rares $(0,5 \mathrm{p}$. Ioo des follicules pileux en activité) pour qu'on ait pu en tenir compte.

Ces mesures ont montré que la vitesse de croissance en longueur s'était trouvée réduite de $60 \pm 2,5 \mathrm{p}$. Ioo et le diamètre de $50 \pm 5 \mathrm{p}$. Ioo par rapport au niveau initial entre le $I 4^{e}$ et le $28^{e}$ jour après l'hypophysectomie (tabl. I et voir fig. 4).

Ainsi, non seulement la production pondérale est diminuée par la mise en repos des follicules pileux, mais encore par la réduction de la production de ceux qui étaient restés encore en activité ; il en résulte une chute d'autant plus brutale de la croissance pondérale : de $60 \pm 7 \mathrm{p}$. IOo à $14 \mathrm{j}$, de $94 \pm 2 \mathrm{p}$. Ioo à $28 \mathrm{j}$ et de Ioo $\mathrm{p}$. Ioo à $42 \mathrm{j}$ après 1'hypophysectomie (tabl. I et voir fig. 4). 


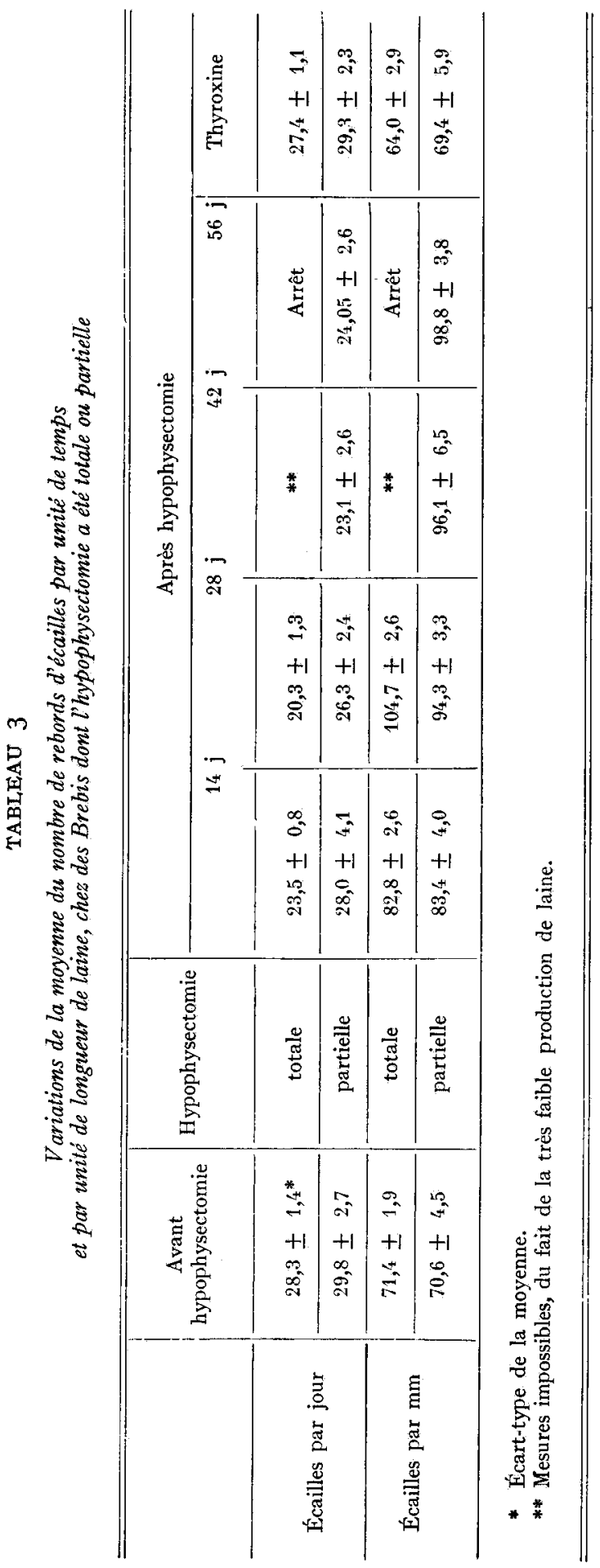


Enfin, le ralentissement de la production des follicules pileux en activité s'est accompagné d'une modification de la structure de la cuticule de la laine (tabl. 3) : le nombre d'écailles produites par jour diminue environ de $28 \mathrm{p}$. Ioo $(20,3 \pm \mathrm{I}, 3$ écailles/jour au lieu de $28,3 \mid \pm \mathrm{I}, 4$ avant l'hyposectomie) mais, comme la vitesse de croissance en longueur s'est réduite dans de fortes proportions, il en résulte que les écailles sont plus serrées les unes par rapport aux autres (ro4,7 $\pm 2,6$ écailles $/ \mathrm{mm}$ au lieu de $7 \mathrm{I}, 4 \pm \mathrm{I}, 9$ écailles/mm avant hypophysectomie).
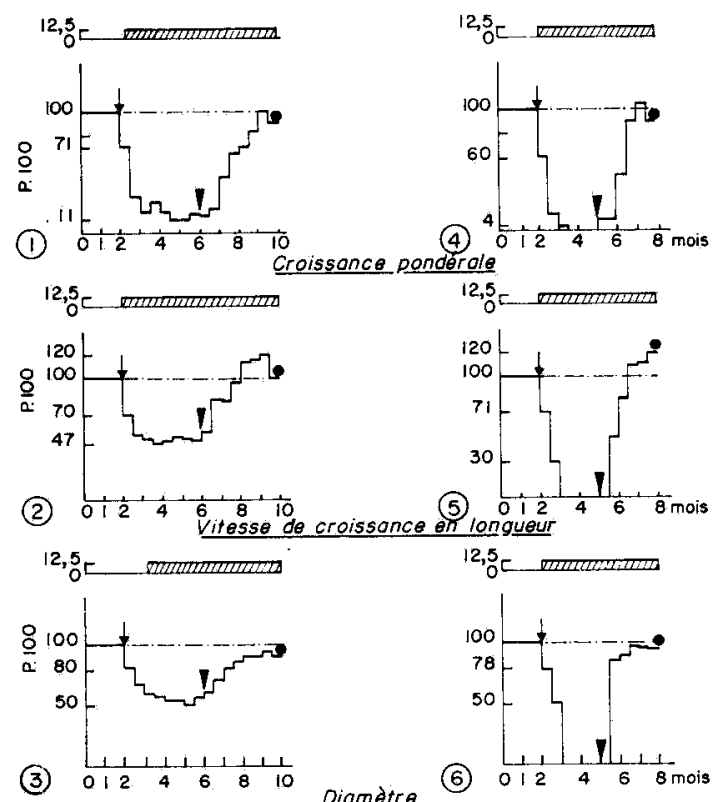

FIG. 4. - Comparaison de la production de laine entre une Brebis incomplètement hypophysectomisée $(\mathrm{I}, 2,3)$ et une brebis dont l'ablation a été totale $(4,5,6)$

légende $\left\{\begin{array}{l}\downarrow \begin{array}{l}\text { date de l'hypophysectomie } \\ \text { date de la I ere injection de L-thyroxine }(6,25 \mu \mathrm{g} / \mathrm{K} / \mathrm{j} \\ \text { arrêt du traitement }\end{array} \\ \text { cortisone }(12,5 \mathrm{mg} / \mathrm{j})\end{array}\right.$

Chez les Brebis, dont 1'hypophysectomie a été partielle, on observe également entre le $\mathrm{I}_{4}$ et le $28^{\mathrm{e}}$ jour, une réduction quoique plus faible de la vitesse de croissance en longueur de la laine $(47 \pm 3,5 \mathrm{p}$. Ioo) et une diminution de son diamètre $(35 \pm \mathrm{r}, 5$ p. Ioo), ce qui expliquerait qu'en dépit d'un nombre important de follicules pileux en activité ( $30 \pm \mathrm{I}, 5$ p. Ioo), la production pondérale de laine est assez faible : I8 \pm 5 p. Ioo du niveau initial (tabl. I et voir fig. 4).

\section{Administration de la thyroxine chez les Brebis dont l'hypophysectomie a été totale}

2. I. Reprise de l'activité des follicules pileux.

Chez les Brebis dont 1 'hypophysectomie a été totale, la $L_{-}$-thyroxine, quelle que soit la dose injectée $(I, 25,6,25, \mathrm{I} 2,50$ et $40,0 \mu \mathrm{g} / \mathrm{kg} / \mathrm{j})$ a rétabli progressivement 


\begin{tabular}{|c|c|c|c|c|c|c|c|c|c|c|c|c|c|c|c|}
\hline & & & $\because$ & $:$ & & & & & & & & & & & \\
\hline & & & 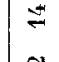 & $\Xi$ & & & & & & & & & & & \\
\hline & & 莺 & & $\stackrel{\approx}{\stackrel{2}{2}}$ & 8 & $\stackrel{\circ}{\sim}$ & 18 & 8 & $\stackrel{\infty}{\infty}$ & $\stackrel{\infty}{=}$ & $\stackrel{\infty}{\circ}$ & ஜ & 5 & $\mid \xi$ & \\
\hline & & 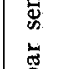 & \& & $\stackrel{8}{\rho}$ & $\stackrel{9}{9}$ & $\stackrel{\circ}{\varrho}$ & $\stackrel{\infty}{\infty}$ & 8 & $\mathscr{8}$ & $\stackrel{*}{*}$ & 8 & 8 & 4 & $\mid \xi$ & \\
\hline క్రు & & $\begin{array}{l}2 \\
0 \\
\text { D. }\end{array}$ & $\infty$ & s & $\bar{\Xi}$ & 苍 & $\stackrel{8}{1}$ & 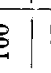 & Бे & $\Xi$ & $\bar{\sigma}$ & $\delta$ & 8 & 18 & \\
\hline & & 苞 & 0 & 挡 & s. & \& & 18 & 穷 & 8 & $\stackrel{2}{z}$ & 8 & $\bar{\infty}$ & $\mid \infty$ & 19 & \\
\hline है & & & ث & $\circ$ & $\infty$ & $\delta$ & 15 & & $\mathscr{\infty}$ & $\stackrel{్}{s}$ & 8 & $\bar{\infty}$ & 19 & $\approx$ & \\
\hline క్ర & & & N & $\circ$ & 草 & 0 & 0 & $b$ & 0 & 10 & 0 & 10 & 10 & 010 & \\
\hline है & & & $\stackrel{2}{2}$ & $s$ & & & & & & & & & & & \\
\hline 2 & &. & $=$ & $\infty$ & & & & & & & & & & & \\
\hline $\begin{array}{l}8 \\
.5\end{array}$ & : & 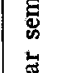 & $\mp$ & $\sigma$ & $\stackrel{ }{=}$ & $\stackrel{*}{\stackrel{*}{*}}$ & $\stackrel{-}{8}$ & 8 & $\stackrel{*}{*}$ & $\stackrel{5}{\sim}$ & $\stackrel{*}{\ominus}$ & $\mid$ & $\stackrel{*}{\stackrel{*}{\sigma}}$ & $\mid \stackrel{*}{\stackrel{*}{*}}$ & \\
\hline & : & $\mid \begin{array}{l}\tilde{z} \\
\underline{z}\end{array}$ & $\stackrel{9}{2}$ & $\stackrel{\infty}{\stackrel{\infty}{n}}$ & 跑 & $\stackrel{*}{\circ}$ & $\stackrel{\oplus}{\rightleftharpoons}$ & E. & $\Xi$ & $\stackrel{2}{\rightleftarrows}$ & $\stackrel{g}{g}$ & ๙ึ & $\stackrel{F}{2}$ & 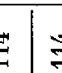 & \\
\hline$\underset{\mathbb{E}}{\mathbb{8}}$ & $\begin{array}{l} \pm \\
\Xi \\
\Xi\end{array}$ & 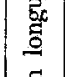 & $\infty$ & $\therefore$ & $\stackrel{2}{9}$ & 8 & $\mid \stackrel{*}{*}$ & 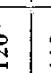 & $\stackrel{\infty}{\Xi}$ & $\stackrel{*}{*} \stackrel{*}{*}$ & $\stackrel{\infty}{\stackrel{0}{*}}$ & 莡 & $\stackrel{*}{*}$ & 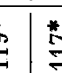 & \\
\hline \& & $\begin{array}{l}\text { 苂 } \\
z\end{array}$ & $\mid \begin{array}{l}0 \\
\ddot{g}\end{array}$ & 10 & 挡 & 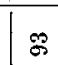 & N & $\stackrel{*}{\stackrel{*}{*}}$ & t. & జ & 芯 & 8 & $\Xi$ & $\stackrel{\infty}{\stackrel{\infty}{\leftrightarrows}}$ & $\xi$ & $E$ \\
\hline है & 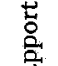 & $\mid \begin{array}{c}.0 \\
0 \\
0\end{array}$ & $\omega$ & 0 & $\ddot{0}$ & tr & $\stackrel{ }{ }$ & & 요 & 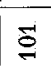 & is & 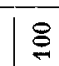 & 18 & 8 & \\
\hline-0 & 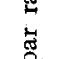 & & or & 0 & 悉 & 10 & 0 & 0 & 0 & 0 & 0 & 0 & 10 & 0 & o \\
\hline हูป & $\S$ & & 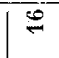 & 18 & & & & & & & & & & & \\
\hline కี & $\begin{array}{l}\dot{a} \\
\dot{\Xi}\end{array}$ & . & 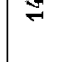 & $\bar{\infty}$ & & & & & & & & & & & \\
\hline$\vec{z}$ & 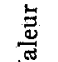 & 袁 & 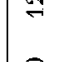 & $\mathscr{\infty}$ & $\dot{\sigma}$ & 8 & $\stackrel{\infty}{\circ}$ & $\infty$ & $\mathscr{8}$ & $\stackrel{\text { s. }}{\sim}$ & 8 & 8 & $\$$ & $\mid$ & $\frac{8}{8}$ \\
\hline हี & & $\begin{array}{l}\frac{\sigma}{2} \\
0 \\
0\end{array}$ & $=$ & $g$ & 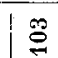 & $\left.\right|_{\infty}$ & ส & S & $\stackrel{80}{g}$ & \pm & $\therefore$ & 16 & 8 & $\mid \vdots$ & $E$ \\
\hline है & & $\frac{\mathrm{g}}{\mathrm{g}}$ & $\infty$ & \& & $\ddot{\infty}$ & 18 & $\stackrel{\circ}{~}$ & 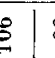 & 8 & 岁 & 18 & 108 & $\infty$ & So & 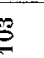 \\
\hline ษ & & $\ddot{g}$ & 0 & 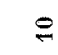 & 8 & in & $\therefore$ & $=$ & $\underset{\sim}{\mathscr{\sigma}}$ & $\Sigma$ & is & $\mathscr{~}$ & ஜ & 8 & \\
\hline . & & 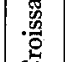 & + & 0 & הి & in & 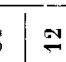 & N & ? & a & $\stackrel{P}{\Gamma}$ & s) & 20 & 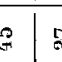 & \\
\hline$\approx$ & & & o & 0 & $\infty$ & 10 & $\infty$ & ○ & $\approx$ & $\stackrel{m}{=}$ & $\stackrel{\circ}{\circ}$ & 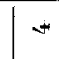 & o & v & 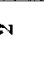 \\
\hline & & $\stackrel{8}{8}$ & & \& & 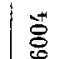 & $\mid \nsubseteq$ & 焉 & 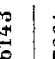 & $\bar{\rho}$ & 吕 & : & 冓 & 윰 & E & 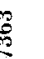 \\
\hline & & 뽀ํ & & - & & & $*$ & & & 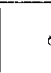 & or & & $\infty$ & 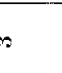 & \\
\hline & & & & 墭 & & & สู & & & 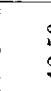 & : & & \& & & \\
\hline
\end{tabular}


l'activité des follicules pileux; en effet, sur les flancs, chez les Brebis recevant de 6,25 à $40,0 \mu \mathrm{g} / \mathrm{kg} / \mathrm{j}$ de $\mathrm{L}$-thyroxine, nous avons observé de 0,5 à $3 \mathrm{p}$. Ioo de follicules pileux en activité $I_{4} j$ après le début du traitement et chez la Brebis recevant la dose la plus faible de $I, 25 \mu \mathrm{g} / \mathrm{kg} / \mathrm{j}$, nous avons noté $I, 5 \pm 0, I$ p. roo de follicules pileux en activité, mais $42 \mathrm{j}$ après le début du traitement, soit avec un retard de 28 jours.

L'activité des follicules pileux n'a été totalement rétablie qu'après 4 mois de traitement : à ce moment $2,5 \pm 0,2$ p. Ioo de follicules pileux étaient au repos, pourcentage à peine plus élevé que celui observé chez les Brebis avant 1'hypophysectomie (moins de I p. Ioo de follicules pileux au repos : tabl. I).

Notons que, lorsque les follicules pileux ont repris leur activité, ils contiennent côte à côte la pointe du nouveau poil de laine en train de se former et la racine de l'ancien poil qui ne va pas tarder à tomber. Le remplacement de la laine s'est donc effectué de la même façon que celui des poils produits par les follicules pileux à fonctionnement cyclique, ce qui confirme donc que les follicules pileux présentaient bien après 1'hypophysectomie le stade normal de repos folliculaire, le télogène.

Quant à la chute de l'ancienne toison, consécutive à la reprise d'activité des follicules pileux, elle ne se produit pas progressivement : au contraire, la toison se détache par plaques entières, lorsque le nombre de poils anciens, ayant quitté le follicule pileux, atteint une certaine proportion, ce qui se produit en moyenne 2 mois après l'administration de L-thyroxine (fig. 5 et 6).

\section{2. Action sur la croissance en longueur (tabl. 4).}

Une fois que le follicule pileux a repris son activité, la vitesse de croissance en longueur du poil de laine qu'il produit se rétablit plus ou moins rapidement suivant les animaux et indépendamment des doses (tab1. 4) : dans la quinzaine qui suit la reprise d'activité des follicules pileux c'est-à-dire au bout de 4 semaines de traitement, elle n'est que de 34 à 75 p. Ioo chez certaines Brebis (n' $6004,7340,7360$ et $736 \mathrm{r}$ ) alors que chez d'autres, elle rétablit le niveau initial (no 6r43, 7338, 7359, 7363).

A la dose la plus faible de $\mathrm{I}, 25 \mu \mathrm{g} / \mathrm{kg} / \mathrm{j}$, la L-thyroxine rétablit à $94 \mathrm{p}$. Ioo le taux moyen normal de la croissance de la laine au bout de I2 semaines de traitement. Mais avec les doses plus élevées $(6,25,12,50$, et $40 \mu \mathrm{g} / \mathrm{kg})$ le niveau normal de la vitesse de croissance en longueur est retrouvé dès la $6^{\mathbf{e}}$ semaine de traitement et même dépassé significativement de Io à 26 p. Ioo par la suite: ainsi, ces doses agissent de façon identique bien que la plus élevée d'entre elles $(40, \mu \mathrm{g})$ soit 6,4 fois supérieure à la plus faible $(6,25 \mu \mathrm{g})$.

\section{3. Action sur le diamètre (tabl. 4).}

Dans la quinzaine qui suit la reprise de l'activité des follicules pileux, les dia mètres moyens de la laine ont déjà presque repris leur valeur normale. Pour la Brebis $\mathrm{n}^{\circ} 4 \mathrm{I} 90$, qui a reçu la dose la plus faible de thyroxine $(\mathrm{I}, 25 \mu \mathrm{g} / \mathrm{kg} / \mathrm{j})$, cela se produit entre $1 \mathrm{a} 6^{\mathrm{e}}$ et $8^{\mathrm{e}}$ semaine de traitement $(92 \mathrm{p}$. 100) et pour les autres Brebis entre la $2^{\mathrm{e}}$ et la $4^{\mathrm{e}}$ semaine ( 73 à $9 \mathrm{I} \mathrm{p}$. IOo), sauf pour la brebis $\mathrm{n}^{0} 7359$, où l'apparition de poils grossiers 'fait dépasser de $7 \mathrm{p}$. Ioo la valeur initiale du diamètre. Par la suite, le diamètre est rétabli à sa valeur initiale, les écarts constatés par rapport à celle-ci n'étant jamais significatifs, sauf pour la Brebis no 7359 (I2I p. IOo).

Ainsi, le diamètre de la laine a repris rapidement sa valeur initiale, indépendamment de la vitesse de croissance en longueur (fig. 4). 


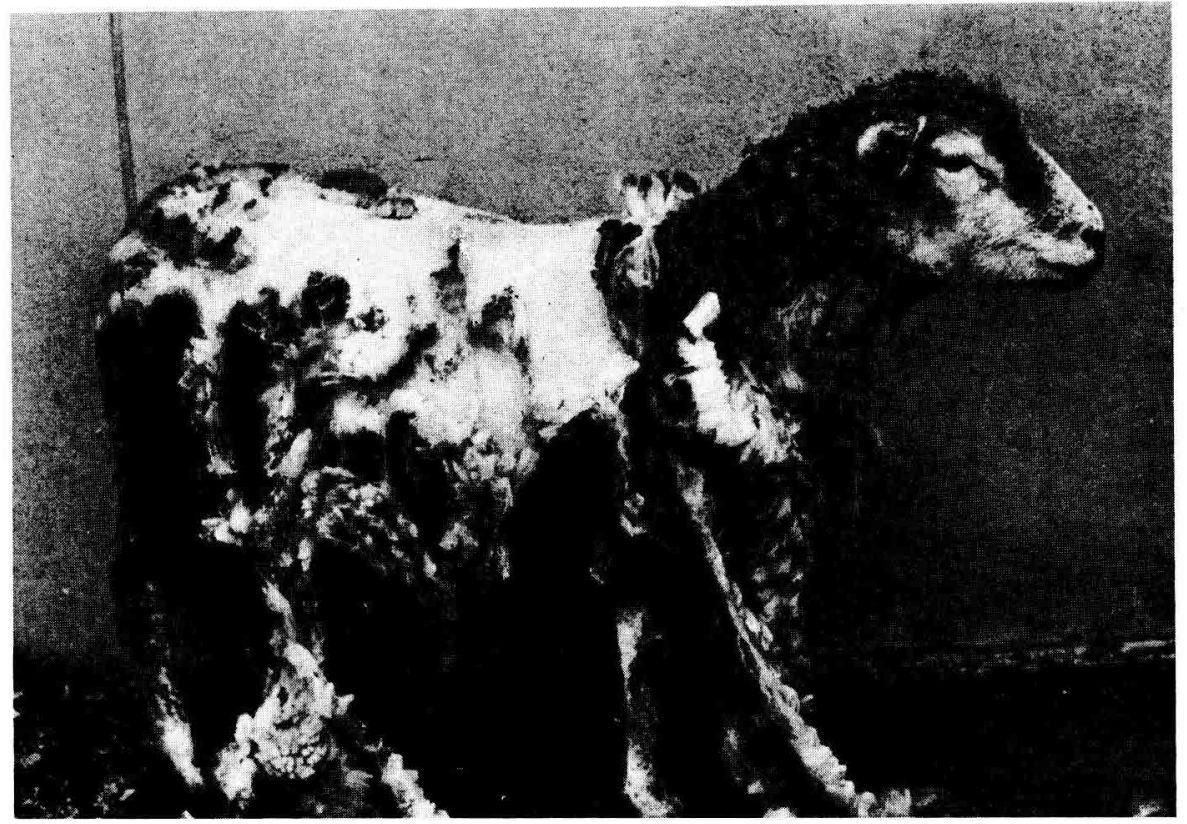

FIG. 5. - Chule de la toison après injection de la inyroxine tl.ez une Brebis hypophyseclomisée

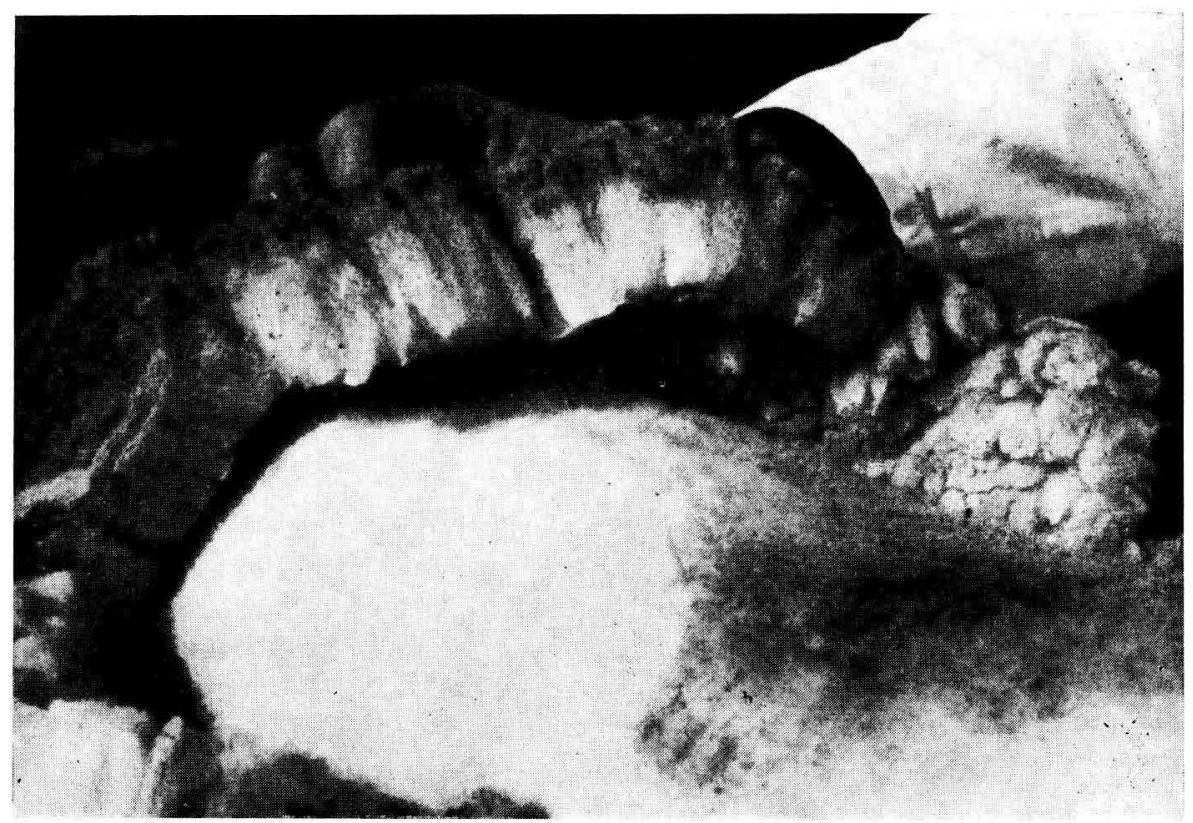

Fig. 6. - Détails de la chute de la toison:

On remarquera sous l'ancienne toison dont la croissance a été complètement arrêtée à la suite de l'hypophysectomie, le nouveau poil de laine dû à l'injection de la thyroxine 
2. 4. Action sur la croissance pondérale (tabl. 4).

La croissance pondérale a été rétablie très progressivement chez tous les animaux et n'a atteint sa valeur initiale qu'au bout de 2 à 3 mois de traitement (ex. fig. 4) ne la dépassant qu'exceptionnellement (pour la Brebis no 7359 où étaient apparus les poils grossiers). Cela est dû au caractère très progressif du rétablissement de l'activité des follicules pileux et, à un degré moindre, de celui de la vitesse de croissance en longueur.

La production en poids de laine a atteint $95 \mathrm{p}$. Ioo de sa valeur initiale, au bout de 16 semaines, à la dose de $I, 25 \mu \mathrm{g} / \mathrm{kg} / \mathrm{j}$ et pour les doses plus élevées, dès la $8^{\mathbf{e}}$ ou ro semaine quelle que soit la dose, de $6,25 \mu \mathrm{g} / \mathrm{kg} / \mathrm{j}$ à 6,4 fois plus.

2. 5. Examen de la cuticule (tabl. 3).

Le nombre de rebords d'écailles produites par jour chez les ro brebis n'est pas significativement différent de celui qui existait antérieurement avant 1'hypophysectomie, la moyenne étant pour le traitement de la thyroxine de $27,4 \pm \mathrm{r}, \mathrm{I}$ écailles par jour au lieu de $28,3 \pm \mathrm{I}, 4$. Quant au nombre d'écailles par mm, il a repris pratiquement sa valeur initiale de $64,0 \pm 2,9$ lorsque la croissance en longueur du brin de laine se rétablissait.

\section{Administration de la thyroxine aux Brebis dont l'hypophysectomie a été incomplète}

Elle présente peu d'intérêt et n'est donnée qu'à titre comparatif (fig. 4).

\section{DISCUSSION}

Après 1'hypophysectomie totale, la croissance de la laine s'arrête pratiquement au bout de 4 semaines. Dans le cas où il subsiste quelques îlots de cellules hypophysaires fonctionnelles, la croissance de la laine diminue très progressivement et si elle s'arrête, cela ne se produit qu'au bout de plusieurs mois seulement. On est donc en droit de penser que c'est là l'explication des résultats de FERGUSON et al. (I965) où la croissance de la laine de certaines Brebis Mérinos hypophysectomisées continue jusqu'à 26 semaines.

Par contre, la présence de la pars tuberalis et de fragments de la tige pituitaire n'ont pas empêché l'arrêt total de la croissance de la laine.

La dose de cortisone de $\mathrm{I} 2,5 \mathrm{mg} / \mathrm{j} /$ Brebis pour maintenir en survie les animaux, après l'hypophysectomie, It'a eu aucune action sur la mise en télogène des follicules pileux. En effet, nous avons vérifié, comme l'avaient fait LINDNER et FERGUson (I956), l'action inhibitrice de la cortisone chez des Brebis intactes : aux doses de $\mathrm{I} 2,5,25$ et $50 \mathrm{mg} / \mathrm{j} /$ Brebis nous n'avons observé aucun effet sur la croissance de la laine ; ce n'est qu'avec Ioo $\mathrm{mg} / \mathrm{j}$ que la croissance pondérale de la laine diminue de $40 \mathrm{p}$. roo.

Les follicules pileux qui ont cessé leur activité après 1'hypophysectomie sont tous en télogène comme l'ont montré, d'une part leurs caractéristiques histologiques et d'autre part le processus de la reprise de croissance de la laine avec deux poils côte à 
côte dans le même follicule. La mise en télogène des follicules pileux producteurs de laine est à souligner car on en a jamais observé plus de $\mathrm{I}$ p. Ioo dans des conditions normales chez les races ovines à toison exclusivement composée de laine (RYDER, I967).

La thyroxine a donc rétabli totalement, à elle seule, la croissance de la laine dans ses dimensions et sa morphologie chez les Brebis hypophysectomisées mais avec des réponses plus ou moins rapides selon les animaux. Par contre, les diverses doses de thyroxine de 6,25 à $40,0 \mu \mathrm{g} / \mathrm{kg} / \mathrm{j}$, n'ont pas eu d'effet différent. Seule la dose de $I, 25 \mu \mathrm{g} / \mathrm{kg} / \mathrm{j}$ a provoqué une réponse nettement plus tardive mais on ne peut tirer de conclusions dans ce cass puisque nous n'avions qu'une seule Brebis ainsi traitée.

Le taux moyen de sécrétion de la thyroxine chez le Mouton, étant de 2,4 à $4,8 \mu \mathrm{g} / \mathrm{kg} / \mathrm{j}$ (HENnEman, REINECKE et GRIFFIN, I955), on peut donc supposer que l'excès de thyroxine est rapidement métabolisé car nous n'avons jamais observé de signes d'hyperthyroïdie. Ces doses sont comparativement plus faibles que celles utilisées par THERIEZ et ROUGEOT (I962) $(50$ à I50 $\mu \mathrm{g} / \mathrm{kg} / \mathrm{j})$ mais, dans ce cas, il s'agissait de DL-thyroxine, donc moins active, et d'administration par voie intraveineuse.

Quand les follicules pileux entrent en activité, après plusieurs semaines de traitement, la vitesse de croissance en longueur de la laine se rétablit rapidement, au cours de la $2^{\mathrm{e}}$ quinzaine qui suit le rétablissement de l'activité folliculaire et peut même dépasser to à 26 p. roo sa valeur normale, à partir de la $3^{\mathrm{e}}$ quinzaine. Quant aux diamètres, ils reprennent plus rapidement leur valeur initiale, soit dès la première quinzaine après la reprise de l'activité folliculaire, et quelle que soit la dose de thyroxine administrée, ne la dépassent jamais significativement.

Ces observations, sur la différence d'action de la thyroxine sur la longueur et le diamètre de la laine concordent avec celles de THERIEZ et RoUGEOT (I962) faites sur la thyroïdectomie et 1'administration consécutive de thyroxine, où seule la longueur de la laine fut affectée et non le diamètre. Cela signifie que la thyroxine n'agit pas sur les dimensions $\mathrm{du}$ follicule pileux, notamment celles du bulbe et de la gaine interne dont dépend le diamètre de la laine, mais sur le métabolisme des cellules du bulbe du follicule pileux en affectant, par conséquent, la vitesse de croissance en longueur de la laine.

En outre, comme dans les expériences de ROUGEOT (I965) on peut dire que la morphologie de la surface de la cuticule de la laine est liée à sa vitesse de croissance en longueur : en effet, l'administration de la thyroxine a rétabli la structure de la surface cuticulaire de la laine qui avait été modifiée à la suite de l'hypophysectomie.

La restauration de la croissance de la laine par la thyroxine seule, pose un problème physiologique si on se réfère à l'effet de 1'hypophysectomie et à celui de la thyroidectomie sur la croissance de la toison : en effet, la thyroïdectomie ralentit seulement la croissance en longueur de la laine, sans affecter le diamètre, et ne supprime jamais 1'activité des follicules pileux. Comment expliquer alors que la thyroxine est capable de rétablir totalement l'activité des follicules pileux chez les Brebis hypophysectomisées?

Ceci conduit à émettre plusieurs hypothèses. Peut-on supposer, comme FERGuson et al. (I965) qu'il existe une source extrathyroïdienne de thyroxine (thyroïde accessoire) dépendante de l'hormone thyréotrope (T. S. H.) ? Pour que cette hypothèse soit valable, il faut supposer l'existence d'une source de thyroxine assez importante pour maintenir après la thyroïdectomie, la croissance de la laine à un taux qui n'est jamais inférieur à 45 p. Ioo du niveau initial ; ce qui semble peu vraisem- 
blable car THERIEZ et Rougeot (I962) ont contrôlé après thyroïdectomie l'absence d'incorporation de ${ }^{131}$ I dans d'éventuelles thyroïdes accessoires.

On peut aussi penser qu'après la thyroïdectomie, l'hypophyse maintient tous les follicules pileux en activité, mais avec un fonctionnement ralenti, par un facteur autre que T. S. H. Ceci revient à dire, que dans le cas de l'hypophysectomie, la thyroxine aurait la potentialité de ce facteur hypophysaire autre que l'hormone thyréotrope. Il reste donc à vérifier l'existence de ce facteur en aảministrant des extraits hypophysaires totaux à des Moutons hypophysectomisés et thyroïdectomisés. S'il y a effectivement action, il s'agira de déterminer alors le facteur responsable.

Reçu pour publication en avril 1969.

\section{SUMMARY}

\section{RESUMPTION OF WOOL GROWTH INDUCED BY THYROXINE INJECTION} IN HYPOPHYSECTOMIZED «ILE-DE-FRANCE 》 EWES

Investigations on weight gain of the wool, length growth and diameter of the fibre, morphology of the cuticle, activity and density of the wool follicles were carried out on I 8 Ile-de-France ewes, whose fleece is made of wool fibres exclusive of any cycle-growing hair.

Three experimental stages were considered :

- before hypophysectomy ;

- after hypophysectomy, with no hormone supplementation apart from a $12.5 \mathrm{mg}$ daily dose of cortisone necessary for the survival of the animals ; roxine.

- after hypophysectomy, with daily injection of $1.25,6.25,12.50,40.0 \mu \mathrm{g}$ per kg of-L-thy-

At the end of the experiments, histological analyses of the sella turcica showed that hypophysectomy had been thoroughly performed in Io ewes, whereas 8 ewes still had clusters of functional pituitary cells.

I. When the hypophysectomy was complete, the activity of the follicles progressively diminished until they showed histological characteristics typical of the resting phase of follicles with a cyclic activity, known as the telogen stage (fig. 3). $5 \pm$ I.5 per cent of follicles had reached this stage at day 7 after hypophysectomy, roo per cent at day 28-42 depending on the animals (table $I$ )

The follicles remained at this stage as long as no treatment was started ( 2 to 12 months depending on the experiment). During this lapse of time, no decrease in the number of follicles was recorded (table 2), and no disappeareance of either sebaceous glands or sweat glands or arrector pili muscles was noticed (fig. 3 ).

Before the follicles were at rest, length growth and diameter of fibres underwent parallel reductions (table I, fig. 4). The morphology of the cuticle was consequently altered and the scale edges overlapped to a greater degree (table 3 ).

The weight gain of wool, depending both upon the number of active follicles and upon the length growth and diameter of wool fibres, sharply decreased down to $4^{\circ} \pm 7$ per cent of the previous rate $o$ to $1_{4}$ days after hypophysectomy, to $6 \pm 2$ per cent at $I_{4}$ to 28 days and to o per cent at 42 days (table I, fig. 4).

2. When functional pituitary cells remain, the activity of the follicles is only partly decreased: 30 I I.5 per cent of follicles remained active 28 days after hypophysectomy, and with some variations, there was no considerable further decrease in the number of active follicles after day 28 . Wool lengh growth curve was plateaued at to to 20 per cent of its normal rate, depending on the animal (tables $1,2,3$, fig. 4).

Therefore, total inhibition of follicular activity is achieved only when the hypophysectomy was complete. In view of these results, it seems that the total cessation of wool growth at day 28 can be taken as a reliable criterion for the effectiveness of the hypophysectomy performed.

L-thyroxine administration induced a progressive resumption of follicular activity, irrespective of the injected dose. This fact indisputably substantiates the contention that, after hypo- 
physectomy, the wool follicles were in a telogen-like resting phase, as the histological picture suggested. Moreover, it is not until the follicular activity is resumed that the old fibres shed and the shedding of the fleece spreads by large patches (fig. 5 and 6).

The follicles in fresh activity grew new fibres with the same characteristics as before hypophysectomy as for diameter and cuticle surface (tables 3 and 4 ); but length growth varied depending on the dose of thyroxine administered. The wool fibres were ro to 20 per cent longer than before hypophysectomy for $6.25, \mathrm{I} 2.5^{\circ}$ and $40.0 \mu \mathrm{g}$ doses, versus 6 per cent shorter for the lowest thyroxine dose, viz. $1.25 \mu \mathrm{g}$ (table 4 , fig. 4).

These findings on the differential action of thyroxine on wool length and diameter are in accordance with previous literature (THERIEZ and ROUGEOT, 1962) on thyroidectomy and subsequent administration of thyroxine.

However, there remains a discrepancy with the investigations cited above insofar as they have shown that thyroxine was only effectual on length growth of the wool, whereas in our experiments on hypophysectomy, thyroxine can induce by itself the resumption of activity of the whole follicle population.

\section{RÉFÉRENCES BIBLIOGRAPHIQUES}

Barrnetr R. J., Seligmann A. M. 1954- Histochemical demonstration of sulfhydryl and disulfide group of proteins. J. Nat. Can. Inst., 14, 769-803.

Carter H. B., 1943. Studies in the biology of the skin and fleece of sheep I. 3. Bull. Coun. Sci. Industr. Res. Aust., 164, 59 pp.

FERGUSON K. A., WALl.ACE L. G., LINDNER H. R., I965. Hormonal regulation on wool growth. In : A. G. LYNE and B. F. SHORT. Biology of the skin and hair growth, 655-677, Angus and Robertson, Sydney.

Fraser A. S., ShORT B. F., I960. The biology of the fleece. Anim. Res. Labo. techni., paper no 3, I-108, CSIRO, Australia.

Henneman H. A., Reinecke E. P., Griffin S. A., I955. The thyroid secretion rate of sheep as affected by season, age, breed, pregnancy and lactation. J.anim. Sci., 14, 419-434.

LINDNER H. R., FERGUSON K. A., 1956. Influence of the adrenal cortex on wool growth and its relation to "break" and "tenderness" in the fleece. Nature, 17\%, 188 -1 89.

RovGEOT J., r959. Mesure de la croissance individuelle des brins de laine à l'aide de la radiocystine. Ann. Zootech., 8, $175^{-1} 77$.

RougEor J., 1962. Étude de la croissance individuelle des brins de laine à l'aide des radio-isotopes. In : International Atomic Energy Agency. The Use of radio-isotopes in animal biology and the medical sciences, Mexico, 1, 199-210, Acad. Press London and N. Y.

ROUGEOT J., I 965 . The effect of thyroid hormones on the morphology of the wool cuticle. In : A. G. LyNE and B. F. SHORT., Biology of the skin and hair growth, 625-640, Angus and Robertson, Sydney.

Ryder M. L., 1967. Wool fibre shedding in some Merino sheep. Aust. J. agric. Res., 18, 683-687.

THERIEZ C., RougEOT J., 1962. Action des hormones thyroïdiennes sur la croissance en longueur du brin de laine." Ann. Biol. anim. Bioch. Biophys., 2, 5-I . 